\title{
Erratum to: "Comparison of intervention effects in split-mouth and parallel-arm randomized controlled trials: a meta-epidemiological study"
}

Violaine Smaïl-Faugeron ${ }^{1,2,3,7^{*}}$, Hélène Fron-Chabouis ${ }^{3,4}$, Frédéric Courson ${ }^{2,3}$ and Pierre Durieux ${ }^{1,5,6}$

\section{Erratum}

The original version of this article unfortunately contained a mistake in the Abstract. The correct information is given below.

"We selected 18 systematic reviews, for 15 meta-analyses with binary outcomes (28 split-mouth and 28 parallel-arm RCTs) and 19 meta-analyses with continuous outcomes (45 split-mouth and 48 parallel-arm RCTs)."

\section{Author details}

Institut National de la Santé et de la Recherche Médicale, U1138, Equipe 22,

Centre de Recherche des Cordeliers, Paris, France. ${ }^{2}$ Assistance

Publique-Hôpitaux de Paris, Hôpital Bretonneau, Service d'Odontologie, Paris,

France. ${ }^{3}$ Université Paris Descartes - Sorbonne Paris Cité, Faculté de Chirurgie

Dentaire, Unité de Recherches Biomatériaux Innovants et Interface, EA4462

Montrouge, France. ${ }^{4}$ Assistance Publique-Hôpitaux de Paris, Hôpital Charles

Foix, Service d'Odontologie, Ivry- sur-Seine, France. ${ }^{5}$ Université Paris Descartes -

Sorbonne Paris Cité, Faculté de Médecine, Paris, France. ${ }^{6}$ Département

d'Informatique Hospitalière, Assistance Publique-Hôpitaux de Paris, Hôpital

Européen Georges Pompidou, Paris, France. ${ }^{7}$ Unité de Recherches Biomatériaux

Innovants et Interface EA4462, 1 rue Maurice Arnoux, 92120 Montrouge, France.

Published online: 02 September 2015

\section{Reference}

1. Smail-Faugeron S, et al. Comparison of intervention effects in split-mouth

and parallel-arm randomized controlled trials: a meta-epidemiological study. BMC Medical Research Methodology. 2014;14:64.

\footnotetext{
* Correspondence: violaine.smail-faugeron@parisdescartes.fr

'Institut National de la Santé et de la Recherche Médicale, U1138, Equipe 22,

Centre de Recherche des Cordeliers, Paris, France

${ }^{2}$ Assistance Publique-Hôpitaux de Paris, Hôpital Bretonneau, Service

d'Odontologie, Paris, France

Full list of author information is available at the end of the article
} 